\title{
Lateral Venorrhaphy for Infrarenal Inferior Vena Cava Injury
}

\author{
Dong Hun Kim \\ Department of Surgery, Trauma Center, Dankook University Hospital, Cheonan, Korea
}

Inferior vena cava (IVC) injuries are extremely lethal with high mortality despite advancing critical care. However, the mortality of IVC injuries usually vary according to the anatomical location of the IVC with infrarenal IVC injuries relatively survivable. Infrarenal IVC is exposed by a right-sided medial visceral rotation, the control of bleeding is initially achieved through manual compression, and then damage control options include ligation, shunt, or repair. Herein we report the use of lateral venorrhaphy of infrarenal IVC injury shown via a video clip.

Key Words: Inferior Vena Cava; Injury; Repair; Compression

(Trauma Image Proced 2017(2):90-91)

\section{CASE}

A 42-year-old woman was admitted with a stab injury to the right abdomen. Upon arrival, the injury site was accompanied with herniation of the omentum and transverse colon (Fig.). The patient underwent emergency laparotomy while evidencing unstable hemodynamics. During the operation, the injury pathway of stab wound was identified to extend from the penetration of the right abdominal wall to the laceration of the right psoas muscle and infrarenal inferior vena cava (IVC) and through the perforation of the proximal transverse colon and mesenteric laceration. The infrarenal IVC injury (2 cm-length laceration) was exposed by a right-sided medial visceral rotation and managed with a lateral venorrhaphy following a proximal and distal control of the injured IVC (Video). Following this, a right hemicolectomy with a double barrel enterostomy was completed and the patient was discharged on hospital day 34.

\section{DISCUSSION}

IVC injury carries a high mortality rate of $65 \%$ with type and anatomical location of the injury, initial severity of shock, and some associated injuries serving as predictive factors for mortality (1). Furthermore, treatment includes damage control options such as ligation, repair, or shunt, which are made intraoperatively, appear critical to patient survival. In the infrarenal IVC injuries, ligation compared with primary repair was associated with poor outcome $(2,3)$. However, for critically injured patient, IVC ligation can be an therapeutic option. Access to the infrarenal IVC is best achieved via a right-sided medial visceral rotation. IVC bleeding can be immediately controlled by direct

Received: September 7, 2017 Revised: September 15, 2017 Accepted: September 21, 2017

Correspondence to: Dong Hun Kim, Department of Surgery, Trauma Center, Dankook University Hospital, 201 Manghyang-ro, Dongnam-gu, Cheonan, Chungnam 31116, Korea

Tel: 82-41-550-7661, Fax: 82-41-550-0039, E-mail: saint7331@gmail.com

Copyright (c) 2017 Korean Association for Research, Procedures and Education on Trauma. All rights reserved.

@This is an open-access article distributed under the terms of the Creative Commons Attribution Non-Commercial License (http://creativecommons.org/ licenses/by-nc/4.0) which permits unrestricted noncommercial use, distribution, and reproduction in any medium, provided the original work is properly cited 


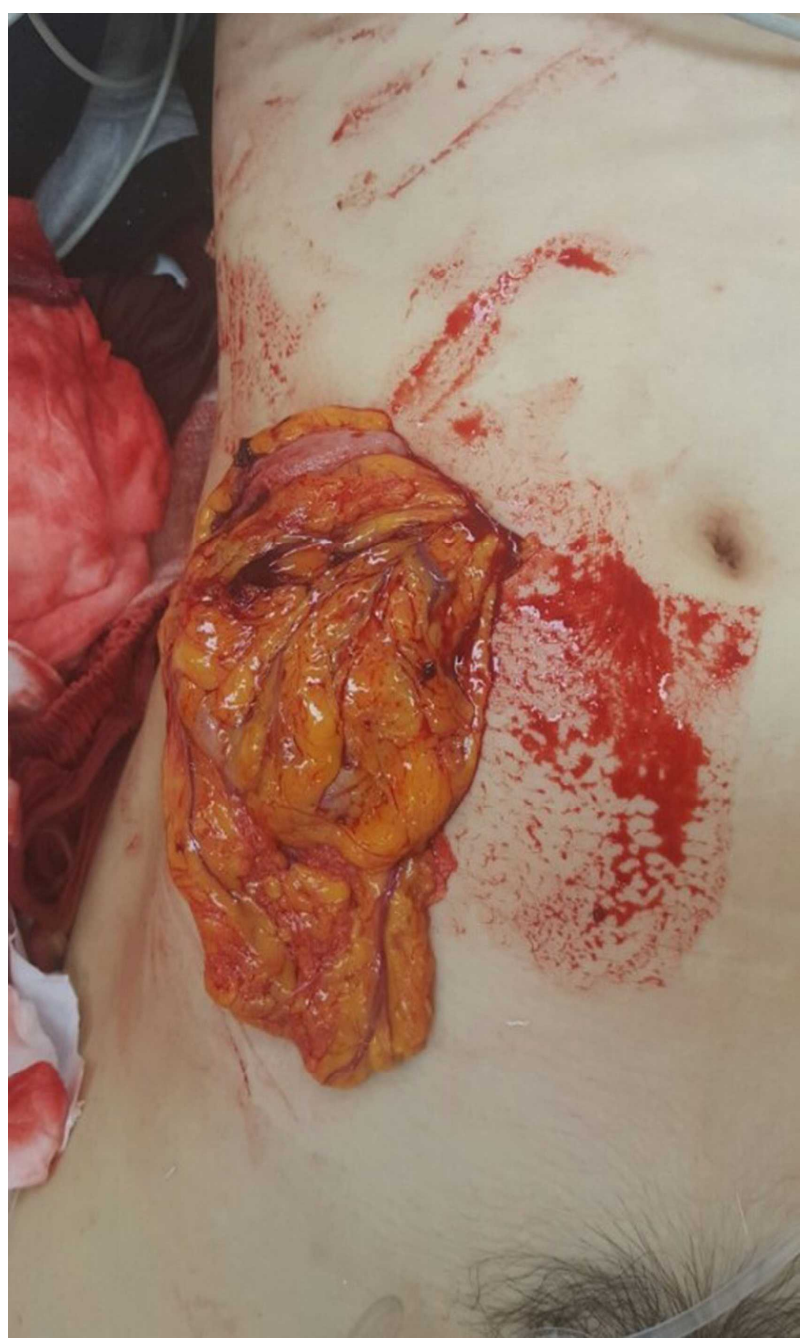

Fig. A photography of the abdomen reveals herniation of the omentum and transverse colon via stab wound.

pressure application against the spine using a swab on a sponge stick at the proximal and distal portion of injured IVC. This control method can facilitate suture repair. A lateral repair of IVC, lateral venorrhaphy, may be rather simple and fast to perform, and most authors recommend the use of simple continuous suture of 4-0 nonabsorbable monofilament polypropylene (Prolene) (4).

\section{Conflicts of Interest Statement}

None of authors have a conflict of interest.

\section{REFERENCES}

1. Rosengart MR, Smith DR, Melton SM, May AK, Rue LW 3rd. Prognostic factors in patients with inferior vena cava injuries. Am Surg. 1999 Sep;65(9):849-55; discussion 855-6.

2. Sullivan PS1, Dente CJ, Patel S, Carmichael M, Srinivasan JK, Wyrzykowski AD, et al. Outcome of ligation of the inferior vena cava in the modern era. Am J Surg. 2010 Apr;199(4):500-6.

3. van Rooyen PL, Karusseit VO, Mokoena T. Inferior vena cava injuries: a case series and review of the South African experience. Injury. 2015 Jan;46(1):71-5.

4. Jonathan LE. Open and endovascular management of blunt and penetrating nonaortic abdominal vascular injury. In: Stanley JC, Veith FJ, Wakefield TW, editors. Current therapy in vascular and endovascular surgery. 5th ed. Philadelphia: Elsevier Saunders, 2014; p.673-4.

\section{Video Legend}

Video. An intraoperative video recording shows inferior vena cava (IVC) exposure with a right-sided medial visceral rotation following temporary control of mesenteric bleeding, and primary repair using 4-0 Prolene suture with proximal and distal compression. 